EPiC Series in Engineering
Volume 3, 2018, Pages 520-525
HIC 2018. 13th International
Conference on Hydroinformatics

\title{
Error propagation from remotely sensed surface soil moisture into soil water index using an exponential filter
}

\author{
Domenico De Santis ${ }^{1}$ and Daniela Biondi ${ }^{1}$ \\ ${ }^{1}$ University of Calabria, Arcavacata di Rende, Italy \\ d.desantis@dimes.unical.it, daniela.biondi@unical.it
}

\begin{abstract}
In this study an error propagation (EP) scheme was introduced in parallel to exponential filter computation for soil water index (SWI) estimation. A preliminarily assessment of the computed uncertainties was carried out comparing satellite-derived SWI and reference root-zone in situ measurements. The EP scheme has shown skills in detecting potentially less reliable SWI values in the study sites, as well as a better understanding of the exponential filter shortcomings. The proposed approach shows a potential for SWI evaluation, providing simultaneous estimates of time-variant uncertainty.
\end{abstract}

\section{Introduction}

Validation and uncertainty characterization of satellite-based soil moisture data constitute fundamental steps to support their suitability for a specific application. An estimate of the root-zone value is required in many of application fields where soil moisture plays a key role, and the exponential filter (Wagner, Lemoine, \& Rott, 1999) is widely used for this purpose, as the method involves only one parameter to obtain a profile soil water index (SWI) from remotely-sensed surface soil moisture (SSM) time series. However, the uncertainty of SWI estimates has been poorly analysed. Among the several methods available for uncertainty assessment, error propagation (EP) schemes have been also developed for remote sensing SSM retrieval algorithms (Naeimi, Scipal, Bartalis, Hasenauer, \& Wagner, 2009). The EP method is based on the propagation of random uncertainties in input variables and model parameters through the model equation, in order to calculate the uncertainty related to each individual model output.

In (De Santis \& Biondi, 2018), a simplified EP scheme aimed at estimating a SWI noise was adapted to exponential filter, which implicitly takes into account both errors and availability of the input SSM data used for each SWI estimation; in this study, SWI uncertainties are estimated by error propagation equations, including the effect of exponential filter parameter uncertainty. The capability 
of EP uncertainty estimates to detect potentially less reliable data in SWI time series is investigated by comparison with available in situ measurements; the role of uncertainties in inputs and in the exponential filter parameter have also been analysed.

\section{Material and methods}

\subsection{Study areas and soil moisture datasets}

In situ soil moisture measurements collected in 10 sites from different networks across Italy and distributed by International Soil Moisture Network (Dorigo, et al., 2011) were used in the following analysis as reference datasets (Table 1). Sites are identified having at least three years of observation during the period 2007-2015, and data available at depths ranging from 20 to $60 \mathrm{~cm}$ were here considered. All sites mainly experience a Mediterranean semi-humid climate. For more details on the in situ dataset see (De Santis \& Biondi, 2018).

\begin{tabular}{llllll}
\hline Code & Station name & Network & Depth $(\mathrm{cm})$ & Observation period & Nearest GPI \\
\hline$\# 1$ & Fitterizzi & Calabria & 30,60 & 1 Jan 2001- 31 Dec 2012 & 2069259 \\
$\# 2$ & Mongrassano & Calabria & 30,60 & 1 Jan 2001- 16 May 2011 & 2069263 \\
$\# 3$ & Torano & Calabria & 30,60 & 1 Jan 2001- 31 Dec 2012 & 2069263 \\
$\# 4$ & Chiaravalle C.le & Calabria & 30,60 & 1 Jan 2001- 31 Dec 2012 & 2034369 \\
$\# 5$ & Satriano & Calabria & 30,60 & 1 Jan 2001-31 Dec 2012 & 2034373 \\
$\# 6$ & Bagnoli & Campania & 30 & 1 Dec 2000 - 21 Nov 2012 & 2128143 \\
$\# 7$ & Cerbara & Umbria & 20,40 & 30 Oct 2009-31 Jul 2014 & 2242295 \\
$\# 8$ & Petrelle & Umbria & 20,40 & 30 Oct 2009-31 Jul 2014 & 2232959 \\
$\# 9$ & Torre Olmo & Umbria & 20,40 & 23 Sep 2009-31 Jul 2014 & 2232971 \\
$\# 10$ & Perugia (Field1) & HYDROL-NET & 25, 35 & 1 Jan 2010-31 Dec 2013 & 2223595 \\
\hline
\end{tabular}

Table 1 Main characteristics of study sites.

The SSM time series derived on a discrete global grid from ASCAT backscatter measurements via the offline WARP processing chain (Naeimi, Scipal, Bartalis, Hasenauer, \& Wagner, 2009) were employed to test the proposed EP scheme, and specifically the DR2016 version distributed as H109 product within the framework of the H-SAF project. ASCAT SSM unit is degree of saturation $S_{d}$ in $\%$, and uncertainty estimates computed by an EP approach are given in the parameter 'soil moisture noise'. The retrieval algorithm is not expected to work properly in areas covered by dense vegetation or characterized by complex topography or open water, and static indicators derived from external datasets can be employed to mask 'unreliable' satellite grid points in operational applications. The proposed SWI error propagation scheme implicitly takes into account situations where poor SSM retrieval performances are expected, being characterised by high values of average 'soil moisture noise' (Naeimi, Scipal, Bartalis, Hasenauer, \& Wagner, 2009). Backscatter measurements from temporary snow covered or frozen land surfaces can also lead to an incorrect determination of SSM. ASCAT SSM dataset includes a surface state flag (SSF): SSM estimates occurring when soil is not unfrozen are masked in SWI estimation. Finally, only data from the ASCAT sensor on board the MetOp-A satellite, covering the period 2007-2015, were here considered.

The nearest neighbour technique was used to match in situ stations with satellite grid nodes (identified by a GPI, Grid Point Index), generally obtaining a single sampling site per grid point. The scale mismatch between satellite footprint and reference point-scale data is a critical aspect, as in situ measurements could show large errors in representing coarse-scale soil moisture. In this sense, data from reference sites have already been used in previous studies aimed at evaluate the satellite-derived 
SWI, e.g. (Brocca, et al., 2011), (Paulik, Dorigo, Wagner, \& Kidd, 2014), (De Santis \& Biondi, 2018). According to the results in (Gruber, Dorigo, Zwieback, Xaver, \& Wagner, 2013), where the coarse scale representativeness of ISMN stations by triple collocation are analysed, "Calabria" sites show lower error values than stations in other considered networks; this is consistent with the better agreement of Calabria network data with the satellite-based SWI found in (De Santis \& Biondi, 2018).

\subsection{Soil water index uncertainty by error propagation}

The root-zone soil water index at time $t_{n}, \mathrm{SWI}_{n}$, can be estimated from previous remotely-sensed surface states at times $t_{i}, \mathrm{SSM}_{i}$, through the exponential filter approach proposed in (Wagner, Lemoine, \& Rott, 1999), that makes use of one parameter only, $T$, representing a characteristic time length. A mathematically equivalent recursive formulation of the exponential filter was proposed in (Albergel, et al., 2008), introducing a gain term, $K_{n}$.

Assuming that errors in SSM measurements and in parameter $T$ are normally distributed and uncorrelated, the uncertainty of $\mathrm{SWI}_{n}$ can be estimated by the error propagation approach as:

$$
\sigma^{2}\left(S W I_{n}\right)=\sum_{i=1}^{n}\left(\frac{\partial S W I_{n}}{\partial S S M_{i}}\right)^{2} \sigma^{2}\left(S S M_{i}\right)+\left(\frac{\partial S W I_{n}}{\partial T}\right)^{2} \sigma^{2}(T)
$$

where:

$$
\begin{aligned}
& \frac{\partial S W I_{n}}{\partial S S M_{i}}=\frac{e^{-\frac{t_{n}-t_{i}}{T}}}{\sum_{i=1}^{n} e^{-\frac{t_{n}-t_{i}}{T}}} \\
& \frac{\partial S W I_{n}}{\partial T}=\frac{1}{T^{2}} \frac{\sum_{i=1}^{n} S S M_{i} e^{-\frac{t_{n}-t_{i}}{T}}\left(\sum_{j=1}^{n} e^{-\frac{t_{n}-t_{j}}{T}}\left(t_{j}-t_{i}\right)\right)}{\left(\sum_{i=1}^{n} e^{-\frac{t_{n}-t_{i}}{T}}\right)^{2}}
\end{aligned}
$$

Equation (1) can be written in recursive form as:

$$
\sigma^{2}\left(S W I_{n}\right)=\Delta_{n}^{2}+\left(\frac{\partial S W I_{n}}{\partial T}\right)^{2} \sigma^{2}(T)
$$

where:

$$
\begin{aligned}
& \Delta_{n}^{2}=K_{n}^{2} \sigma^{2}\left(S S M_{n}\right)+\left(1-K_{n}\right)^{2} \Delta_{n-1}^{2} \\
& \frac{\partial S W I_{n}}{\partial T}=\frac{K_{n}}{T}\left[G_{n}\left(S W I_{n-1}-S W I_{n}\right)+e^{-\frac{t_{n}-t_{n-1}}{T}} \frac{T}{K_{n-1}} \frac{\partial S W I_{n-1}}{\partial T}\right]
\end{aligned}
$$

having defined $G_{n}$ as:

$$
G_{n}=e^{-\frac{t_{n}-t_{n-1}}{T}}\left(G_{n-1}+\frac{1}{K_{n-1}} \frac{t_{n}-t_{n-1}}{T}\right)
$$

Initial value $\Delta_{0}$ is set to $\sigma\left(\mathrm{SSM}_{0}\right)$, while $\left(\partial \mathrm{SWI}_{0} / \partial T\right)$ and $G_{0}$ are set to 0 . 
The EP scheme implicitly takes into account the effect of possible prolonged temporal gaps in input SSM data, related on the $T$ value.

The term $\Delta$, that considers only SSM uncertainties, is equivalent to the SWI noise index calculated in (De Santis \& Biondi, 2018). The jacobian term ( $\partial \mathrm{SWI} / \partial T)$ assumes high values proportionally to the latest SSM inputs variability on a time-scale related to the $T$ parameter, which reflects in SWI value significant changes, e.g. state transition from dry to wet conditions.

\section{Results and discussion}

SWI time series were computed from SSM datasets, estimating the optimal $T$ value that maximizes the correlation coefficient $R$ between in situ and satellite-based soil moisture observations; after linearly rescaling SWI with the mean and variance matching technique, the root-mean-squaredifference (RMSD) was calculated. Overall, the comparison between SWI and in situ measurements gave good results; the exponential filter generally confirmed a greater ability in capturing the seasonal soil moisture behaviour rather than short time-scale fluctuations.

In parallel SWI uncertainties time series were estimated with the approach described above, considering the $\sigma(\mathrm{SSM})$ provided in ASCAT products and assuming $\sigma(T)$ equal to the $10 \%$ of the estimated $T$ value (Figure 1). As shown in Figure 1, the differences in computed uncertainty due to the introduction of the term related to the parameter $T$ can be remarkable.

It is worth noting that input SSM uncertainties show much lower absolute values and considerably reduced seasonal patterns in this updated ASCAT data records release, compared to previous product versions. The latest product version benefits from several updates, mostly in instrument calibration, model parameters, and algorithms, consequently, no prominent seasonal patterns are reflected in estimated SWI uncertainty time series, as it happened instead in (De Santis \& Biondi, 2018).

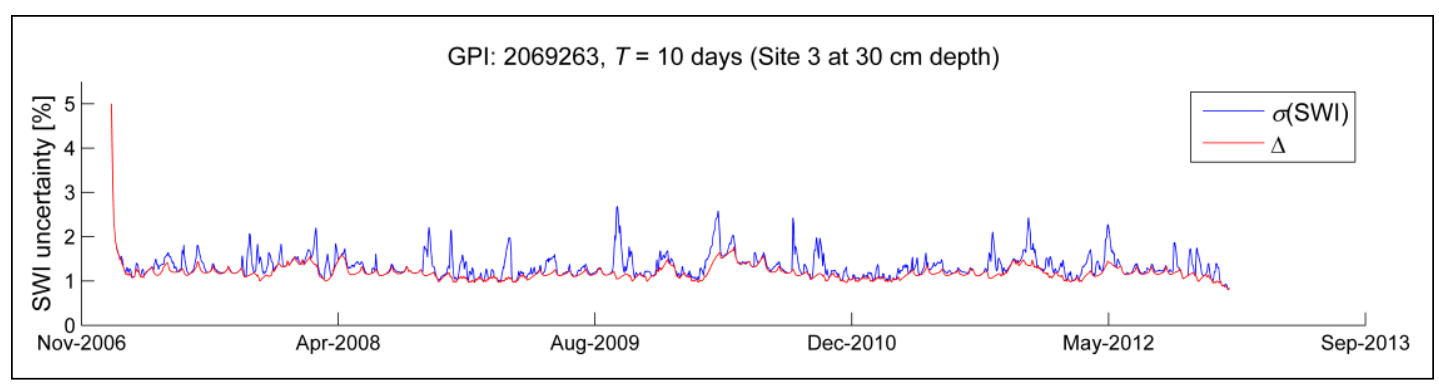

Figure 1 Example of estimated SWI uncertainties time series (in red the values calculated by considering only input uncertainties).

In this study, a preliminarily assessment of estimated SWI uncertainties was carried out, by focusing only on the highest values in the time series, investigating the correspondence with potentially less reliable SWI data, i.e. with data that do not fits well in situ observations. The correspondence between observed deviations and SWI random error realizations theoretically holds well as long as the latter is the main error term. Ground measurements, used as reference for the evaluation of coarse-scale SWI, also contain random errors (instrumental and representativeness); furthermore, residual systematic differences are to be expected, as mean and variance matching technique constitutes a suboptimal rescaling solution (Yilmaz \& Crow, 2013).

The more uncertain SWI values, are firstly masked by setting four $\sigma(\mathrm{SWI})$ thresholds to remove fixed percentage of data $(5,10,15$ and $20 \%)$ and then a linear scaling was performed to remaining SWI data; finally, performance metrics $R$ and RMSD are recomputed to check whether the discarded 
data contributed positively or not to the overall agreement between the in situ and satellite-based soil moisture time series. To examine the role played by parameter uncertainty, the same procedure was also performed by applying thresholds on $\Delta$ values. An example of the obtained results is shown in Figure 2, for 3 representative sites.

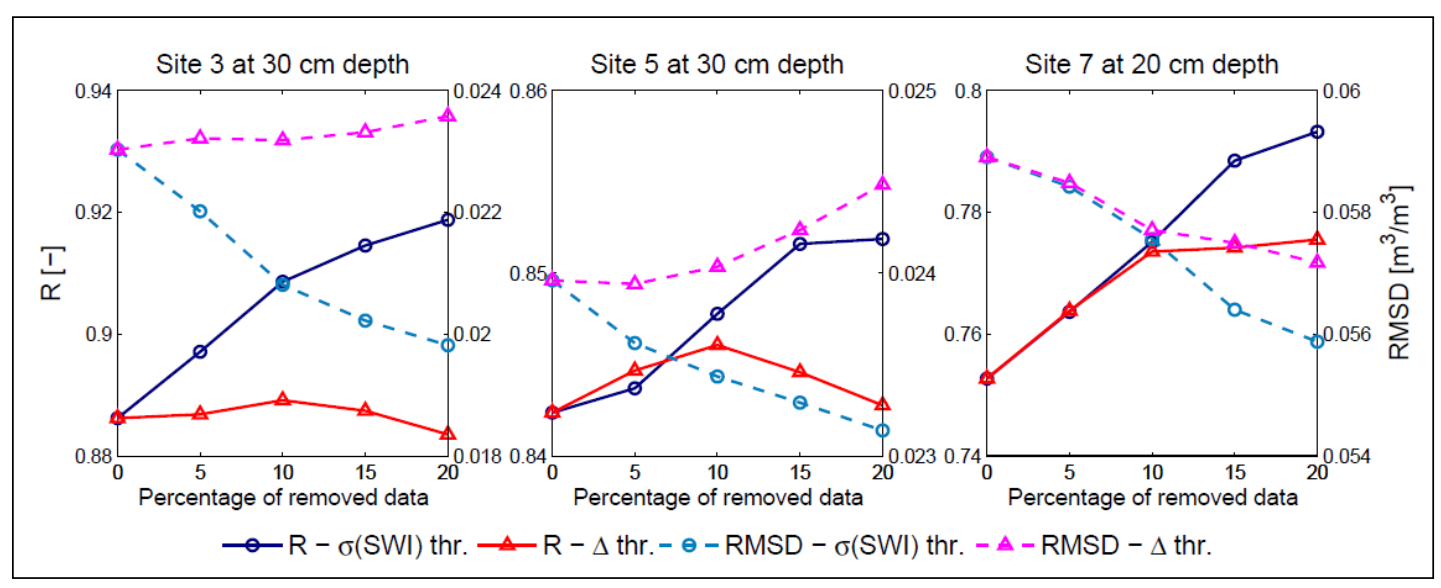

Figure 2 Effects of the different uncertainty thresholds on $R$ and RMSD values, for 3 representative sites.

Overall, the analysis shows a correspondence between the removing more uncertain SWI values and the improving the observed performance metrics. Increasing thresholds on $\sigma(\mathrm{SWI})$ seem to lead to a systematic better agreement between remaining SWI data and reference ground measurements, both in terms of $R$ and RMSD. Conversely, regarding $\Delta$, data masking shows absent or reduced performance improvements, often limited to the removal of a moderate percentage of SWI values (that include those corresponding to exponential filter initializations). It is also noted that masked uncertain SWI data are deemed to be those characterized by high $(\partial \mathrm{SWI} / \partial T)$ values, i.e. significant change in soil moisture value, and thus the performance improvements can be explained by the removal of points that testify the limited skills of exponential filter in detecting short time-scale fluctuations. In this sense, the EP scheme succeeds in identifying SWI estimates that do not capture properly soil moisture state transition and deviate considerably from reference ground measurements.

\section{Conclusions}

Although requiring further research, the proposed EP scheme has shown capabilities to identify potentially less reliable SWI values in the selected study sites, and the preliminary results suggest its utility in the SWI evaluation, in the comprehension of the exponential filter shortcomings, and in providing simultaneous estimates of time-variant SWI uncertainty.

\section{References}

Albergel, C., Rudiger, C., Pellarin, T., Calvet, J.-C., Fritz, N., Froissard, F., et al. (2008). From nearsurface to root-zone soil moisture using an exponential filter: an assessment of the method based on in-situ observations and model simulations. Hydrol. Earth Syst. Sci., 12:6, 13231337. 
Brocca, L., Hasenauer, S., Lacava, T., Melone, F., Moramarco, T., Wagner, W., et al. (2011). Soil moisture estimation through ASCAT and AMSR-E sensors: an intercomparison and validation study across Europe. Remote Sens. Environ., 115:12, 3390-3408.

De Santis, D., \& Biondi, D. (2018). A quality assessment of the soil water index by the propagation of ASCAT soil moisture error estimates through an exponential filter. Int. J. Remote Sens., 39:1, 232-257.

Dorigo, W., Wagner, W., Hohensinn, R., Hahn, S., Paulik, C., Xaver, A., et al. (2011). The International Soil Moisture Network: a data hosting facility for global in situ soil moisture measurements. Hydrol. Earth Syst. Sci., 15:5, 1675-1698.

Gruber, A., Dorigo, W., Zwieback, S., Xaver, A., \& Wagner, W. (2013). Characterizing coarse-scale representativeness of in-situ soil moisture measurements from the International Soil Moisture Network. Vadose Zone J., 12:2.

Naeimi, V., Scipal, K., Bartalis, Z., Hasenauer, S., \& Wagner, W. (2009). An improved soil moisture retrieval algorithm for ERS and METOP scatterometer observations. IEEE T. Geosci. Remote, 47:7, 1999-2013.

Paulik, C., Dorigo, W., Wagner, W., \& Kidd, R. (2014). Validation of the ASCAT Soil Water Index using in-situ data from the International Soil Moisture Network. Int. J. Appl. Earth Obs., 30, $1-8$.

Wagner, W., Lemoine, G., \& Rott, H. (1999). A method for estimating soil moisture from ERS scatterometer and soil data. Remote Sens. Environ., 70:2, 191-207.

Yilmaz, M., \& Crow, W. (2013). The optimality of potential rescaling approaches in land data assimilation. J. Hydrometeorol., 14, 650-660. 